\title{
Leserbefragung
}

\section{Antibiotika bei Atemwegsinfekten unpopulär}

\author{
Der Herbst bringt wie in jedem Jahr eine regelrechte Welle an Patienten mit Infekten der \\ Atemwege in die Praxen. Die Hälfte erwartet laut einer Leserbefragung eine AU-Bescheini- \\ gung. Medizinisch versorgt werden sie demnach primär mit pflanzlichen Arzneimitteln.
}

— Während der Erkältungszeit kommen die meisten Patienten wegen Atemwegsinfektionen zum Arzt. In der Hälfte der Praxen sind es 25-50\% der Patienten, in jeder fünften Praxis sind es sogar über 50\%. Diese Quoten ergeben sich aus der Leserumfrage „Antibiotika-Resistenzen - wie vermeiden?“, die Springer Medizin mit Unterstützung des Unternehmens Bionorica SE im April 2015 durchgeführt hat. Gefragt nach den Erwartungen dieser Patienten, kreuzte rund jeder zweite der fast 240 Teilnehmer "AU-Bescheinigung“ an. Oberster Patientenwunsch ist allerdings die rasche Symptomlinderung (84\%) gefolgt von „schnell wieder leistungsfähig sein“ und der Verordnung eines wirksamen Arzneimittels (beide 57\%).

Der Patientenwunsch nach einem Antibiotikum wird meist nicht erfüllt: Nur $11 \%$ der Ärzte gaben an, bei Atemwegsinfekten ein Antibiotikum zu verordnen (Abb. 1). Zwei Drittel raten dagegen zu pflanzlichen Arzneimitteln. Es folgen der Rat, kürzer zu treten, sowie die Empfehlung unterstützender Maßnahmen, etwa reichlich zu trinken und zu inhalieren (je 61\%).

Antibiotika werden eher gezielt verordnet: $80 \%$ der Ärzte verschreiben sie ihren Risikopatienten, etwa jenen mit COPD oder Immunschwäche. Auch bei schwerer Symptomatik über einen längeren Zeitraum verordnen 71\% Antibiotika. Bei alten, multimorbiden Patienten tun dies $42 \%$. Bei Patienten mit grünlich-gelbem Auswurf sind es 38\%, auch wenn laut einer Studie nur jeder sechste von ihnen tatsächlich eine bakterielle Infektion haben dürfte [Scand J Prim Health Care. 2009;27:70].

\section{CRP-Wert als beliebter Marker}

In dem freien Antwortfeld wurde oft ein hoher CRP-Wert als Indikator für eine Antibiotikaverordnung genannt. Einige Ärzte machen einen CRP-Schnelltest direkt in der Praxis.

Die Teilnehmer sollten auch erklären, wie sie gegenüber den Patienten gegen Antibiotika argumentieren. Dafür gab es

\section{Abb. 1 Ergebnis der Leserumfrage von Springer Medizin: Phytotherapeutika} sind für viele Ärzte das Mittel der Wahl bei Atemwegsinfekten

Frage: Was verordnen Sie Ihren Patienten bei Atemwegsinfekten?

Phytotherapeutika

Ruhe/kürzer treten

Unterstützende Maßnahmen

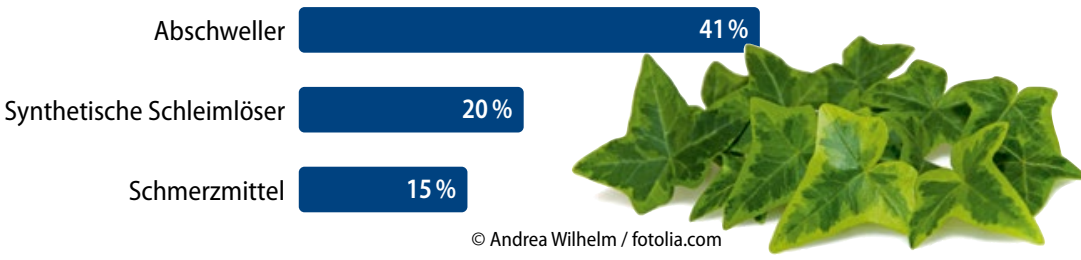

ein freies Antwortfeld. Hier wurde ein Kernargument immer wieder genannt: Antibiotika bringen nichts bei Virusinfekten - Atemwegsinfekte sind meist viral verursacht. Oft wird von den Ärzten auch auf Nebenwirkungen und Resistenzbildung hingewiesen.

\section{Örtliche Resistenzsituation kennen}

Über die Resistenzsituation vor Ort bzw. in der Region wissen rund 30\% der Teilnehmer nicht gut Bescheid. Andere informieren sich bei ihrem Mikrobiologielabor (37\%), beim Robert-Koch-Institut (RKI, 23\%), bei örtlichen Kliniken oder dem Gesundheitsamt (jeweils knapp $10 \%$ ) und den Referenzzentren (6\%). Das Mikrobiologielabor, mit dem eine Praxis zusammenarbeitet, sollte die erste Anlaufstelle für Infos zur Resistenzsituation vor Ort sein. Auch die Antibiotika Resistenz Surveillance (ars) am RKI ist eine geeignete Informationsquelle (https://ars.rki.de).

Viele Ärzte befürworten Bedarfsrezepte für Antibiotika. In der Praxis allerdings nutzt jeder zweite Teilnehmer (47\%) diese Möglichkeit nur selten, jeder vierte (27\%) sogar niemals. $14 \%$ gaben an, häufig Bedarfsrezepte zu nutzen, und nur knapp 5\% berichteten von einem regelmäßigen Gebrauch. Dabei zeigt die Erfahrung, dass ein solches Rezept geeignet ist, den Patienten mit Atemwegsinfekten zusätzlich Sicherheit zu geben. Es dient als Rückversicherung für den Patienten - oder auch, im Falle von Kindern, für die Eltern. So ist bereits nach dem ersten Arztbesuch ein Rezept verfügbar, falls das Labor (Blutbild, BSG, CRP) doch auf einen bakteriellen Infekt hinweist oder „red flags“ wie steigendes Fieber oder Husten mit starken retrosternalen Schmerzen auftreten.

Dr. Michael Hubert 